\title{
Treatment of experimental herpes simplex keratitis with acycloguanosine
}

\author{
D. J. BAUER, ${ }^{1}$ P. COLlins, ${ }^{1}$ W. E. TUCKER, JR., ${ }^{2}$ AND A. W. MACKLIN ${ }^{2}$ \\ From the ${ }^{1}$ Wellcome Research Laboratories, Beckenham, Kent, and ${ }^{2}$ Burroughs Wellcome Co., \\ Research Triangle Park, North Carolina, USA
}

SUMMARY Acycloguanosine, a recently developed compound with high inhibitory activity against viruses belonging to the herpes group, has been evaluated in experimental herpes simplex keratitis in rabbits in comparison with trifluorothymidine and preparations of idoxuridine and vidarabine at present in clinical use. All compounds were used in the form of ophthalmic ointments which were applied 5 times a day at intervals of 2 hours. Treatment began on the third day of infection and was continued for 4 days. Complete cure was obtained with acycloguanosine and idoxuridine; trifluorothymidine and vidarabine were considerably less effective. Acycloguanosine was equally effective when given intravenously in the form of its sodium salt, and could be detected in the tear fluid in inhibitory concentrations when given by mouth. The compound was relatively free from toxicity.

The use of specific antiviral agents is an established form of treatment for herpes simplex keratitis, but in many cases treatment is ineffective or gives rise to hypersensitivity and toxic manifestations. These have been summarised by McGill et al. (1976). Treatment with idoxuridine may cause contact dermatitis, punctate epithelial keratopathy, follicular conjunctivitis, and thickening of the lid margins associated with stenosis or occlusion of the punctum. Trifluorothymidine may cause contact dermatitis, punctate epithelial keratopathy, and oedema of the corneal epithelium. The same signs of toxicity have been observed with vidarabine, and there is a further report that trifluorothymidine may cause filamentary keratosis (Coster et al., 1976). These drugs also have the disadvantage of not being effective by systemic routes of administration.

The present paper describes a new compound, acycloguanosine, having a number of favourable properties which may make it a useful addition to the range of drugs at present available for the treatment of herpes simplex keratitis.

Acycloguanosine [9-(2-hydroxyethoxymethyl)guanine] is an acyclic nucleoside possessing high inhibitory activity against certain viruses of the herpes group, including herpes simplex and varicellazoster. Its antiviral activity and general properties

Address for reprints: Dr D. J. Bauer, Wellcome Research Laboratories, Langley Court, Beckenham, Kent BR3 3BS
Table 1 Relative potencies of acycloguanosine and standard antiherpes simplex compounds

\begin{tabular}{lll}
\hline Compound & $E D 50(\mu M)$ & Relative potency* \\
\hline Acycloguanosine & 0.1 & 10 \\
Idoxuridine & 1.0 & 1 \\
Trifluorothymidine & 1.5 & 0.67 \\
Vidarabine & 16.0 & 0.06 \\
\hline
\end{tabular}

*Relative to idoxuridine $=1$

have been described by Schaeffer et al. (1978), and are summarised in Tables 1 and 2, which include comparative data for 3 other compounds used in the treatment of herpes simplex keratitis. The antiviral activities were determined by means of plaque reduction dose-response lines carried out with a type 1 strain of herpes simplex virus in VERO cell cultures. From these lines are obtained the values for the ED50, which is the concentration of compound in $\mu \mathrm{M}$ which will reduce the number of plaques to $50 \%$ of that in an untreated control preparation. In Table 1 it will be seen from the ED50 values that acycloguanosine is 10 times as active as idoxuridine, 16 times as active as trifluorothymidine, and 160 times as active as vidarabine against the strain of virus used. It therefore has a considerable advantage over these other compounds in respect of its higher antiviral activity. 
Table 2 Properties of acycloguanosine and standard antiherpes simplex compounds

\begin{tabular}{|c|c|c|c|c|}
\hline & Acycloguanosine & Idoxuridine & Trifluorothymidine & Vidarabine \\
\hline Aqueous solubility (\%) & $0 \cdot 14$ & 0.8 & $\geqslant 5$ & 0.05 \\
\hline Stability of solution at $\mathrm{pH} 7$ & Stable & Stable & Unstable & No information \\
\hline Metabolites & Essentially unmetabolised & Inactive & Inactive & Less active \\
\hline Enters aqueous & Yes & Yes & Yes & Yes* \\
\hline Crosses blood-brain barrier & Yes & No & No information & Yes \\
\hline
\end{tabular}

*As metabolite with reduced activity

General properties and some aspects of the clinical pharmacology of the compounds are compared in Table 2. Low solubility is a disadvantage except in the case of trifluorothymidine, but acycloguanosine forms a sodium salt with an aqueous solubility of $2 \%$ which is suitable for intravenous injection. It is catabolised only to the extent of less than $5 \%$, so that practically all the compound is present as the active form. It is present in the blood and urine after oral administration in laboratory animals and human volunteers, and can be found in the tear fluid after intravenous and oral administration of the sodium salt. One hour after oral administration to a dog in a dose of $25 \mathrm{mg} / \mathrm{kg}$ acycloguanosine was present in the aqueous in a concentration 20 times greater than the $100 \%$ inhibitory concentration as established with type 1 herpes simplex virus in tissue culture (de Miranda et al., 1978).

These generally favourable properties make acycloguanosine a suitable candidate for clinical trial, and as a preliminary its antiviral effect was evaluated in rabbits infected on the cornea with herpes simplex virus, and the results are described in the present work as a comparative study with trifluorothymidine and commercially available ophthalmic preparations of idoxuridine and vidarabine. Also described are the results of preclinical toxicity tests in which the ocular safety of acycloguanosine was evaluated in several species of animals treated topically and by parenteral routes of administration.

\section{Materials and methods}

EXPERIMENTAL HERPES SIMPLEX KERATITIS Ophthalmic preparations

In initial experiments acycloguanosine was administered as aqueous suspensions in concentrations ranging from 0.25 to $1 \%$. Benzalkonium chloride was added in a concentration of $0.01 \%$ as a preservative. Subsequently, a series of ointments was prepared in soft paraffin ophthalmic base, in which the concentration of acycloguanosine ranged from 0.03 to $3 \%$. These formulations were prepared in the Wellcome Pharmaceutical Research and Development Laboratories, Greenville, NC, USA.

Idoxuridine was tested in the form of Kerecid, a $0.5 \%$ ophthalmic ointment marketed by Smith, Kline and French Laboratories, Welwyn Garden City, Herts, England.

Vidarabine (ara-A) was used in the form of Vira-A, a 3\% ophthalmic ointment marketed by Parke, Davis \& Co., Ann Arbor, Michigan, USA.

\section{Intravenous preparation}

Ampoules containing $500 \mathrm{mg}$ of acycloguanosine in the form of the freeze-dried sodium salt were supplied by the Wellcome Pharmaceutical Research and Development Laboratories, Greenville, NC, USA. The material was re-dissolved by the addition of $25 \mathrm{ml}$ of sterile distilled water. A fresh ampoule was reconstituted for each series of injections.

\section{Animals}

The experiments were carried out on New Zealand white and half-lop rabbits weighing about $2 \cdot 25 \mathrm{~kg}$. They were obtained from Ranch Rabbits, Crawley Down, Sussex, England. The half-lop rabbits were used in experiments requiring repeated intravenous injections.

\section{Virus}

The work was carried out with the PH8 strain of type 1 herpes simplex virus, which was obtained from Mr J. McGill, Southampton Eye Hospital, Southampton, England. The virus was grown in cultures of VERO cells. At the height of infection the cultures were disrupted by sonication. The stock suspension of virus thus obtained was placed in ampoules in volumes of $1 \mathrm{ml}$ and stored at $-70^{\circ} \mathrm{C}$. For the infection of rabbits an ampoule was thawed and diluted to a concentration of virus of around $10^{6}$ plaque-forming units per millilitre. The same stock of virus was used for all the experiments described.

\section{Method of infection}

Both eyes of a rabbit were infected by a modification 
of the method of Jones et al. (1968) described by Collins and Bauer (1977). The virus was inserted into the corneal epithelium at 8 sites by trephination with a glass capillary tube. The same concentration of virus was used for all insertions. After 3 days the developing lesions were visualised with sodium fluorescein (Fluorets, Smith \& Nephew Pharmaceuticals Ltd., Welwyn Garden City, Herts, England). The extent of the ulceration was recorded by drawings on a protocol sheet, and the degree of infection was quantitated by the numerical scoring method of Corwin et al. (1963).

\section{Treatment}

In all cases treatment was begun on the 3rd day of infection. A drop of acycloguanosine solution was applied to 1 eye 5 times a day at intervals of 2 hours. The other eye was left untreated. Ointments were applied to 1 eye with a similar treatment schedule. The amount of ointment used at each dosing ranged in length from 10 to $15 \mathrm{~mm}$ and weighed 20 to $30 \mathrm{mg}$. The other eye was treated with ophthalmic ointment base for control purposes. Treatment was carried out for 4 days. The extent of infection in both eyes was recorded daily, and the experiments were terminated on the 7th day of infection, at which time the result of treatment was assessed.

The sodium salt of acycloguanosine was injected into the marginal ear vein in a dose of $50 \mathrm{mg} / \mathrm{kg}$. The injections were given twice daily at 9 a.m. and 4 p.m. for 4 days. The result was assessed on the 7th day of infection in comparison with a control group of infected animals which did not receive treatment.

\section{Experimental design}

Preliminary experiments to establish the antiviral activity of acycloguanosine supensions were carried out under open conditions without the use of a placebo. Comparison of acycloguanosine ointments with the other antiviral preparations was carried out by a double-blind procedure with ophthalmic ointment base as placebo, in which the observer was unaware of the treatment given.

Determination of acycloguanosine levels in tear fluid For the determination of acycloguanosine levels a biological method based on the plaque inhibition test (Herrmann, 1961) was used. Discs of filter paper $6 \mathrm{~mm}$ in diameter were placed in the lower fornix of each eye of an animal treated with acycloguanosine and left until saturated with tear fluid. Monolayers of VERO cells in $60-\mathrm{mm}$ plates were infected with type 1 herpes simplex virus and covered with an agarose overlay. A disc was placed in the centre of the overlay and the plates were incubated to allow plaques to develop. The cultures were then fixed with $10 \%$ formaldehyde in phosphate-buffered saline for 1 hour. The overlay was removed and the plaques were visualised by staining the cell sheet with $0.5 \%$ methyl violet in $20 \%$ methanol. The diameter of the zone of inhibition of plaque formation was measured in millimetres. The concentration of acycloguanosine was then read off from a standard curve obtained with discs dipped in solutions of acycloguanosine of known micromolarity. The diameter of the zone of inhibition had a linear regression on the logarithm of the concentration over the range from 5 to $500 \mu \mathrm{M}$.

\section{PRECLINICAL TOXICITY STUDIES Animals}

Evaluation of eye irritancy and ocular toxicity was carried out in New Zealand white rabbits (Franklin Farms, Wake Forest, NC, USA), pure-bred beagle dogs (Marshall Research Animals, North Rose, NY, USA), Sprague-Dawley rats (Charles River Laboratories, Wilmington, Mass., USA) and female CD-1 mice (Charles River Laboratories).

\section{Examination of the eye}

The eyes of all animals were evaluated by indirect ophthalmoscopy, biomicroscopy, and histological examination except where otherwise indicated. Before examination the pupils were dilated with $1 \%$ tropicamide solution ( $1 \%$ Mydriacyl, Alcon Laboratories Inc., Fort Worth, Texas, USA). In eye irritancy studies the cornea was stained with fluorescein (Fluor-I-strip, Ayerst Laboratories Inc., New York, NY, USA). At the end of the test period the animals were killed and the eyes were removed and fixed in Zenker's acetic acid solution, and processed essentially in accordance with the methods described by Levy et al. (1965). Full anteroposterior sections approximately $7 \mu \mathrm{m}$ thick were taken through the optic nerve head, stained with haematoxylin-eosin, and examined by conventional light microscopy.

\section{STUDIES}

Eye irritancy study in rabbits

Groups of 8 rabbits were treated with 1, 3, and $6 \%$ ointments of acycloguanosine in white petrolatum vehicle. Control groups were dosed with vehicle or balanced saline. The test materials were instilled into the conjunctival sac of 1 eye of each rabbit 5 times daily at 90 -minute intervals for 21 consecutive days. The untreated eye served as an additional control. The condition of the eyes was assessed numerically by the method of Draize (1959) 5 days and 1 day before beginning treatment, and on 3 rabbits of each group on treatment days $1,2,4,7$, 14 , and 21. Examination with the biomicroscope and 
ophthalmoscope was carried out 7 days before treatment, on the last day of the treatment period, and finally 22 days later. Five rabbits in each group were killed at the end of the 21-day dosing period and the remaining 3 per group 24 days later. Both eyes of each animal were removed and examined histologically.

\section{Intravenous study in dogs}

Groups of 8 beagle dogs were given intravenous injections of the sodium salt of acycloguanosine in doses of $10,20,25$, and $50 \mathrm{mg} / \mathrm{kg}$ twice daily for 30 consecutive days. It was prepared as an isotonic solution in sterile $0.4 \%$ sodium chloride solution. Two control groups of 8 dogs were dosed with $0.9 \%$ sodium chloride solution. The interval between doses was about 6 hours. The eyes were examined before and at the end of dosing. Histological examination was carried out on the eyes of all dogs except for those in the lowest dosage group.

\section{Intravenous study in rats}

Groups of 15 male and 15 female rats were given single daily intravenous injections into the tail vein of the sodium salt of acycloguanosine in doses of 20 , 40 , and $80 \mathrm{mg} / \mathrm{kg}$ for 21 days. A control group was treated similarly with $0.9 \%$ sodium chloride solution. Examination of the eye was carried out on all animals before dosing and on 10 males and 10 females of each group on the 16th day of dosing. Histological examination was carried out on both eyes of all rats in the highest dosage group and of all animals in the control group.

\section{Oral study in mice}

Groups of 38 male and 38 female mice were given acycloguanosine in $0.25 \%$ agar by stomach tube in daily doses of 50,150 , and $450 \mathrm{mg} / \mathrm{kg}$ for 33 days. A control group was given agar alone. Histological examination was carried out on 10 males and 10 females randomly chosen from the top dosage group and the controls. The eyes were not examined by other methods.

The opportunity was taken to obtain additional data on ocular toxicity in animals used for the teratology studies described below.

\section{Teratology study in rats}

Groups of 21 to 25 pregnant rats were given acycloguanosine subcutaneously in doses of $6,12.5$, and $25 \mathrm{mg} / \mathrm{kg}$ twice daily from the 6th to the 15 th days of gestation inclusive. Controls were given $0.9 \%$ sodium chloride solution. The eyes of 8 rats in the control and highest dosage groups and 11 each from the other 2 dosage groups were examined before and

\begin{tabular}{|c|c|c|c|c|c|}
\hline $\begin{array}{c}\text { DAY OF } \\
\text { INFECTION }\end{array}$ & 3 & 4 & 5 & 6 & 7 \\
\hline $\begin{array}{c}\text { DAY OF } \\
\text { TREATMENT }\end{array}$ & 1 & 2 & 3 & 4 & - \\
\hline $\begin{array}{c}\text { CONTROL } \\
\text { EYE }\end{array}$ & $\ddots$ & & - & & \\
\hline $\begin{array}{c}\text { TREATED } \\
\text { EYE }\end{array}$ & $\vdots$ & $\ddots$ & & & \\
\hline
\end{tabular}

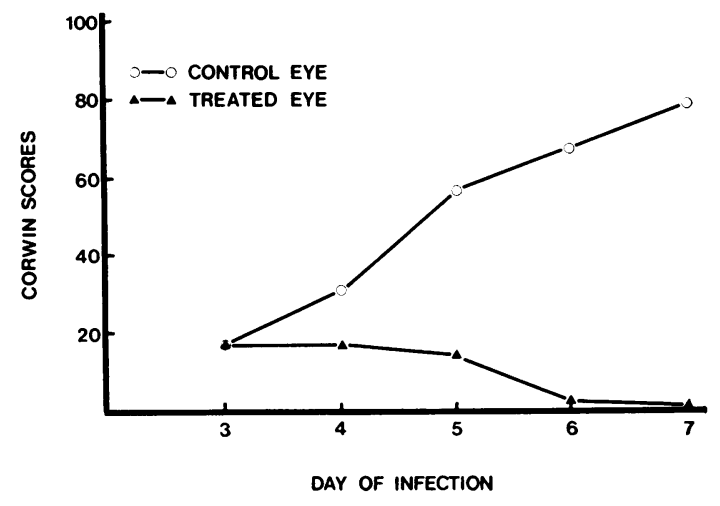

Fig. 1 Completed protocol form of a rabbit treated with $1 \%$ acycloguanosine eye-drops. Shaded areas indicate corneal ulceration

after dosing. On the 20th day of gestation the same animals were killed and the eyes were examined histologically.

Teratology study in rabbits

Groups of 16 to 18 pregnant rabbits were treated from the 6th to the 18th days of gestation inclusive with the same dose levels used in the rat study. The eyes were examined before treatment and on the 29th day of gestation. The animals in the control and highest dosage groups were killed on the 29th day and the eyes were examined histologically.

In a repetition of the study carried out for the purpose of increasing the number of litters available for examination observations of the eyes were limited to visual examination.

\section{Results}

ACYCLOGUANOSINE EYE DROPS

In preliminary experiments to evaluate antiviral activity aqueous suspensions of acycloguanosine were tested in 10 rabbits. Cure was not always obtained with the 0.25 and $0.5 \%$ suspensions over the 4-day period of treatment, but a satisfactory effect was obtained with the $1 \%$ suspension. The results of such an experiment are shown in Fig. 1, 
Fig. 2 Effects of treatment with a range of acycloguanosine ointments, Vira- $A$, trifluorothymidine, and Kerecid on herpes simplex keratitis. Acycloguanosine. $\square$ Vira-A.

$\diamond$ Trifluorothymidine. $\triangle$ Kerecid. Control

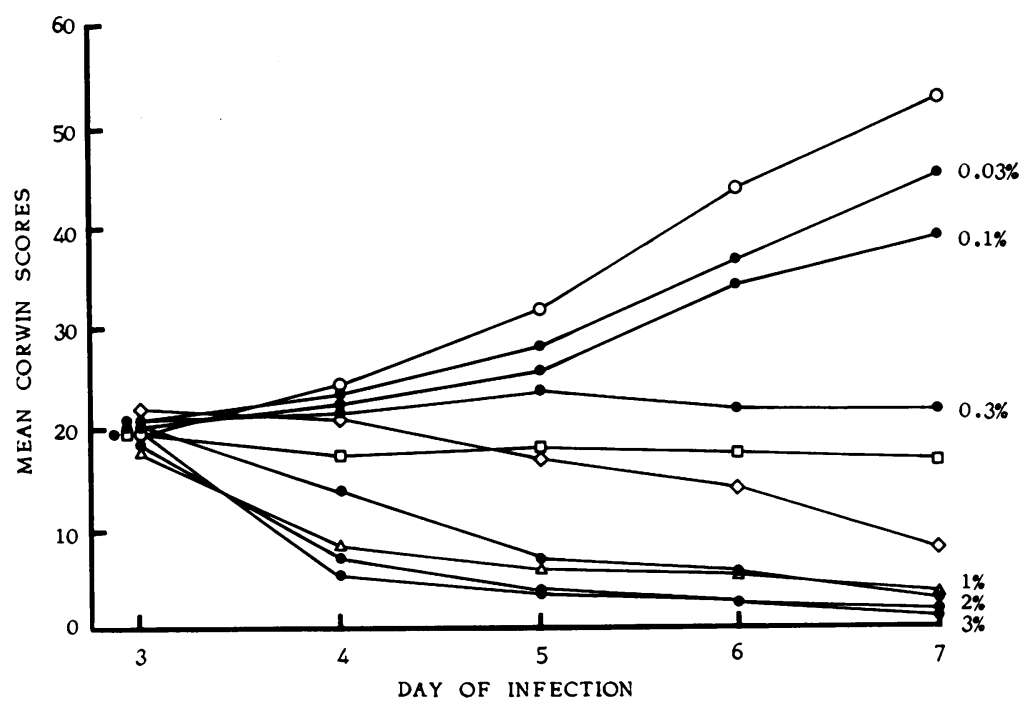

which illustrates the correspondence between the extent of the corneal lesions and the graphs of the Corwin scores. Healing is complete by the day after the termination of treatment and the score has fallen to zero, whereas in the control eye the ulceration has involved the greater part of the cornea and the score is still increasing.

ACYCLOGUANOSINE OPHTHALMIC OINTMENTS In a similar dose-ranging study ophthalmic ointments containing acycloguanosine in concentrations ranging from 0.03 to $3.0 \%$ were tested in a total of 60 rabbits, 10 rabbits being used for each preparation. The mean Corwin scores for each group of 10 are shown in Fig. 2. The $0.03 \%$ ointment had a perceptible effect in retarding the course of infection. This inhibitory effect increased with rising concentration, and with the $1 \%$ preparation cure was practically complete by the 4th day of treatment. The 2 and $3 \%$ ointments were both somewhat more effective, giving the maximum curative effect with the treatment regimen employed.

\section{COMPARISON OF ACYCLOGUANOSINE WITH} KERECID, VIRA-A, AND TRIFLUOROTHYMIDINE In similar experiments Kerecid and Vira-A were each tested in 10 rabbits and trifluorothymidine in 8. The graphs of mean Corwin scores for each preparation are included in Fig. 2. From this it will be seen that cure by the 4th day of treatment was obtained only with acycloguanosine and Kerecid. Treatment with trifluorothymidine prevented the further extension of the ulceration, but the lesions were considerably slower in clearing up. Vira-A was less effective still.
ACYCLOGUANOSINE SODIUM SALT

In an investigation of the antiviral effect of acycloguanosine when administered by the intravenous route in the form of its sodium salt 6 rabbits were infected with type 1 herpes simplex virus in the usual way. Three were treated with the sodium salt by the schedule given in the Methods section, and the other 3 were left untreated as controls. The individual Corwin scores of the 6 rabbits are shown in Fig. 3. Each point represents the mean score for the 2 eyes of 1 animal. Arrest of the infection is evident on the 2 nd day of treatment. After the end of treatment on the 7th day of infection a few punctate areas staining with fluorescein could still be observed. The treated animals were therefore kept for another 3 days and re-examined. The corneas were completely healed with no sign of relapse of the infection. The animals were kept for 12 months and examined at weekly intervals. No evidence of recurrence was observed.

DETERMINATION OF LEVELS OF

ACYCLOGUANOSINE IN THE TEAR FLUID

The levels of acycloguanosine in the tear fluid of a rabbit after a single intravenous dose of $100 \mathrm{mg} / \mathrm{kg}$ are shown in Table 3. The level at 60 minutes is 100 times the $100 \%$ inhibitory concentration as determined by the plaque reduction method in VERO cells. Although the dose was higher than that used in the therapeutic experiment, the cure obtained with intravenous injections of $50 \mathrm{mg} / \mathrm{kg}$ may be assumed to be due to the presence of acycloguanosine in the tear fluid.

Intravenous injection was selected for the purpose of confirming that herpes simplex keratitis could be 


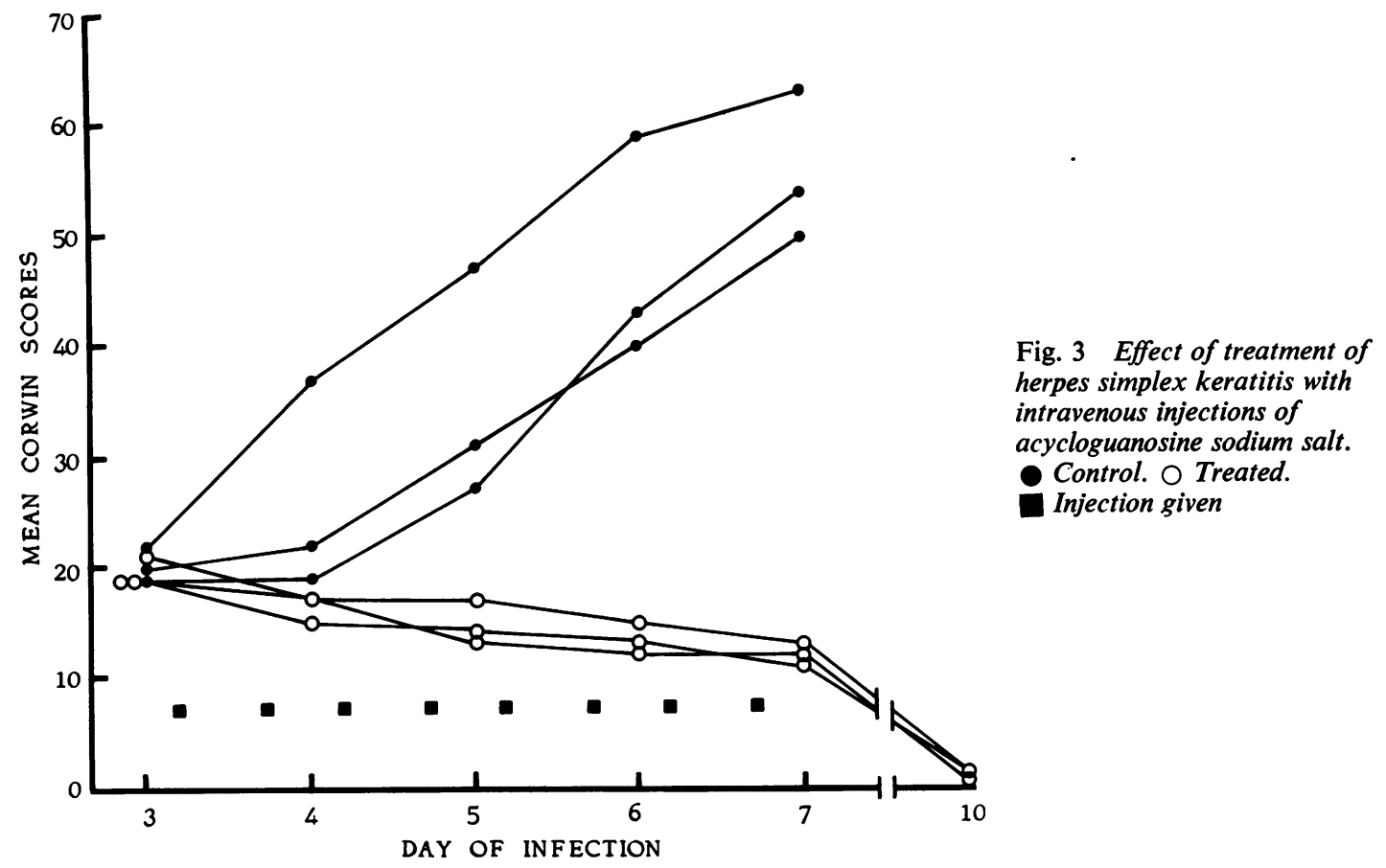

treated successfully by a systemic route. A more practical route in clinical practice would be administration by mouth. A rabbit was therefore given $100 \mathrm{mg} / \mathrm{kg}$ of the sodium salt of acycloguanosine by mouth, and the levels in the tear fluid were determined at intervals up to 240 minutes after administration. The results are given in Table 3. It will be seen that effective levels were present at 30 minutes, and acycloguanosine was still present at $\mathbf{4 0}$ times the $100 \%$ inhibitory concentration after 4 hours.

\section{TOXICITY OF ACYCLOGUANOSINE FOR THE} EYE ON TOPICAL ADMINISTRATION

In the eye irritancy study no signs of irritation occurred in rabbits treated with balanced saline. Rabbits treated with the vehicle and the ointment preparations had minimal signs of irritation which were limited to the conjunctiva and seen only during

Table 3 Levels of acycloguanosine in the tear fluid after systemic administration

\begin{tabular}{lllllllr}
\hline & \multicolumn{8}{c}{ Time (minutes) } \\
\cline { 2 - 7 } Dose $(\mathrm{mg} / \mathrm{kg})$ & Route & 15 & 30 & 40 & 60 & 120 & 240 \\
\hline 100 & Intravenous & $12 *$ & 18 & 29 & 31 & ND & ND \\
100 & Oral & ND & 16 & ND & 14 & 13 & 12
\end{tabular}

- Concentration of acycloguanosine $(\mu \mathrm{M})$. ND = not done

the dosing period. These signs usually appeared at the end of the dosing day and had disappeared by the following morning. The mean Draize scores for rabbits treated with the vehicle and the $1 \%$ ointment were similar. Animals treated with higher concentrations of acycloguanosine had only slightly higher scores. None of the methods of examination revealed any significant potential for ocular toxicity.

TOXICITY OF ACYCLOGUANOSINE FOR THE EYE ON PARENTERAL ADMINISTRATION In none of the studies in which acycloguanosine was given parenterally was there ophthalmological or histological evidence of an effect on the eye. Corneal opacification was observed in 1 rabbit given the highest dose in the second teratology study. It appeared in 1 eye on the 8th day of gestation after 2 days of dosing and was bilateral by the 16th day. The opacities persisted until the animal was killed on the 29th day. Oedema of the outer third to half of the cornea was the only morphological change observed on histological examination. The aetiology was not determined, but it was not considered to be related to treatment with acycloguanosine. No similar lesions occurred in the rabbits in the first teratology study, in pregnant rats treated similarly, or in non-pregnant dogs, mice, and rats given much higher and even lethal parenteral doses of acycloguanosine for longer periods. 


\section{Discussion}

The results show that satisfactory cure of herpes simplex keratitis in rabbits can be obtained with 1,2 , and $3 \%$ ointments of acycloguanosine, and with Kerecid. The $1 \%$ ointment of trifluorothymidine was less effective, and a complete cure was not obtained with Vira-A over the 4-day period of treatment. It is possible that a better result might have been obtained with Vira-A if it had been given over the daily 15 -hour dosage period recommended by the manufacturers.

The finding of effective levels of acycloguanosine in the tear fluid for at least 4 hours after oral administration suggests that this route may prove effective in clinical use if adequate levels can be obtained in man. Oral administration may be the method of choice for the treatment of herpes simplex keratitis in patients with unimpaired lacrimal function, since it should be more effective in giving sustained levels in the tear film than the intermittent administration of eye drops or ointment. In this respect animal experiments have shown that acycloguanosine has an advantage over the other compounds, which are effective only when given topically.

The possibility of systemic treatment, high intrinsic activity, and lack of toxicity to the eye suggest that acycloguanosine should be of value in the treatment of herpes simplex keratitis in man.

We are very grateful to Mr J. McGill (Southampton Eye Hospital) and Drs G. B. Elion and H. J. Schaeffer (Burroughs Wellcome Co., Research Triangle Park, North Carolina) for valuable comments on the manuscript, and to $\mathrm{Mr} \mathrm{N}$. M. Oliver for technical assistance. Ophthalmological examinations in the toxicology studies were carried out by Drs S. A. Koch, G. L. Blanchard, and W. P. Keller.

\section{References}

Collins, P., and Bauer, D. J. (1977). Comparison of activity of herpes virus inhibitors. Journal of Antimicrobia! Chemotherapy, 3, Supplement A, 73-81.

Corwin, M. E., Coleman, V., Riegelman, S., Okumoto, N., Jawetz, E., and Thygeson, P. (1963). Effect of IUdR and amethopterin on experimental herpes simplex keratitis. Investigative Ophthalmology, 2, 578-583.

Coster, D. J., McKinnon, J. R., McGill, J., Jones, B. R., and Fraunfelder, F. T. (1976). Clinical evaluation of adenine arabinoside and trifluorothymidine in the treatment of corneal ulcers caused by herpes simplex virus. Journal of Infectious Diseases, 133, Supplement A, 173177.

De Miranda, P., Krasny, H. C., and Elion, G. B. (1978). Metabolic disposition and pharmacokinetics of 9-(2hydroxyethoxymethyl)guanine, a new antiviral drug. Abstract No. 902, Abstracts of the 7th International Congress of Pharmacology IUPHAR, July 16-21, Paris, 35.

Draize, J. H. (1959). Dermal toxicity in appraisal of the safety of chemicals in foods, drugs and cosmetics, 46-59. Association of Food and Drug Officials of the United States, Austin, Texas.

Herrmann, E. C (1961). Plaque inhibition test for detection of specific inhibitors of DNA containing viruses. Proceedings of the Society for Experimental Biology and Medicine, 107, 142-145.

Jones, B. R., Wise, J. B., and Patterson, A. (1968). The measurement of enhancement or inhibition of virus replication in the cornea. Evaluation of Drug Effects in the Eye, pp. 83-97. Symposium of the Royal Society of Medicine. Edited by P. V. Pigott. Association of Medical Advisers to the Pharmaceutical Industry: London.

Levy, M. C., Covatta, T. J., Morris, C., and Aschner, H. H. (1965). Technique for preparing histologic sections of dogs' and rabbits' eyes in paraffin. Archives of Ophthalmology, 73, 122-123.

McGill, J., Fraunfelder, F. T., and Jones, B. R. (1976). Current and proposed management of ocular herpes simplex. Survey of Ophthalmology, 20, 358-365.

Schaeffer, H. J., Beauchamp, L., de Miranda, P., Elion, G. B., Bauer, D. J., and Collins, P. (1978). 9-(2-Hydroxyethoxymethyl)guanine activity against viruses of the herpes group. Nature, 272, 583-585. 